\title{
ANNUAL URBAN BUILT-UP CHANGE AREA ONLINE EXTRACTION USING LANDSAT TIME SERIES DATA
}

\author{
Jun Zhang ${ }^{1}$, Hao $\mathrm{Wu}^{1}{ }^{1}$, , Cai Cai ${ }^{2}$ \\ ${ }^{1}$ National Geomatics Center of China, Beijing, 100830, China - (junzhang, wuhao)@ngcc.cn \\ ${ }^{2}$ Beijing Key Laboratory of Urban Spatial Information Engineering, Beijing Institute of Surveying and Mapping, China - \\ caicai361448336@126.com
}

Commission IV, ICWG IV/III

KEY WORDS: Information extraction, Online processing, Urban built-up area, Time series trajectory model, Landsat

\begin{abstract}
:
Urban built-up area change information in multiple periods is a pivotal factor in global climate change application and sustainable development research. Due to spatial-temporal expression of land cover types, processing speed and operability, built-up area change information extraction using Landsat time series data is still a challenging task. To provide insights into the inter-annual dynamic of land use change, focusing on how time series characteristics improves recognition of urban change and how much online extraction convenience is facilitated, this paper presents a new methodology to built-up change area extraction using inter-annual time series of Landsat images. The central premise of the approach is that time series characteristics are firstly expressed by spectral index. The logistic algorithm is then used in time series trajectory modelling of land cover types for annual urban built-up change area extraction. Finally, the individual steps of the whole process, including image selection, time series trajectory modelling and results display, are converted to web service for online processing. The further comparison is also conducted between the proposed method and postclassification comparison method. Results show that the online processing mode has strengths regarding the provision of functionality to user-end, the automation of recurring tasks or the sharing of workflows. Results also demonstrate that the proposed method improves the accuracy of annual urban built-up change area extraction.
\end{abstract}

\section{INTRODUCTION}

Urban built-up area change information in multiple periods (i.e. three or more) is pivotal to understand the complex drivers and mechanisms in global climate change and to forecast future land use trends in sustainable development (Arsanjani, et al., 2016; Aburas, et al., 2018 ). Over the past three decades, urban area in developing countries such as China and India, has been expanding at an unprecedented pace, due to significant economic development and human activities. One consequence is that urban extent will undoubtedly continue to grow, as well as its effect.

In recent ten years, many studies on urban area and change extraction with Landsat time series data have already been carried out (Landsat time series data refers to annual or monthly Landsat images, or all available Landsat images during a long period time like ten or more years). Most existing urban land change studies only use few multi-temporal Landsat data (e.g. images of each five years) in urban expansion analysis. Few existing studies adopted the post-classification method for extraction of annual urban expansion from Landsat time series data, which first classifies Landsat images of each year separately and then compares classification results to obtain urbanization dynamic (Zhang, et al., 2016; Sumari, et al., 2017; Yu, et al., 2018).

Time series analysis algorithm is another existing way for land cover change extraction, in which each original image of time series is firstly expressed as feature image by the feature or index that reflect characteristics and difference among land cover types, feature time series is constructed with these feature images. Thus, pixel-based change trajectory in time series can be obtained and analysed to extract change information of the land cover type (Sun, et al., 2017; Shen, et al., 2017; Quan, et al., 2018). Most existing studies of urban change extraction with time series analysis algorithm focus on vegetation/forest, such as VCT (vegetation change tracker) algorithm (Li, et al., 2009), temporal segmentation algorithm (Cohen, et al., 2010), sin and cos fitting algorithm (Zhu, et al., 2014). Post-classification methods and time series analysis algorithms are efficient approaches for land cover change extraction. However, post-classification methods have high requirement of classification accuracy of each image in time series. If a large classification error exists, it will cause large change extraction error and the subtle changes may not be detected. Because of strong spectral heterogeneity of urban builtup area in such $30 \mathrm{~m}$ spatial resolution images, it is difficult to select or make a factor to construct feature time series for builtup area, which may affect the accuracy of urban built-up area change extraction.

In the intelligent era, the pattern of remotely sensed information processing is gradually transformed from traditional desktopbased geoprocessing to distributed online geoprocessing (Guo, et al., 2010; Wu, et al., 2015). Web-based service has become one of the major solutions for providing online remote sensed processing (Sun, et al., 2012; Chen, et al., 2015). However, most of present studies have focused on online remote sensed image pre-processing (Hofer, et al., 2015), few of online change extraction has been investigated.

\footnotetext{
* Corresponding author
} 
Therefore, in this paper, we proposed a new methodology of mapping urban built-up area growth dynamics from Landsat time series data. All annual Landsat time series images were expressed by one spectral index as a time series feature image. Then the logistic algorithm is used in time series trajectory modelling of land cover types for annual urban built-up change area extraction. The whole process of the extraction is converted to web service for online processing. The proposed method is further compared with the post-classification comparison method. The performance of annual urban built-up change area extraction is assessed.

\section{METHODOLOGY}

The main idea can be summarized as follows. First, one feature factor was selected to extract time series feature image from Landsat time series data (section 2.1). Then time series trajectory from feature image is consistency checked and fitted with the logistic regression model, a time series trajectory model for detecting annual urban built-up change is constructed. The annual urban built-up change area is extracted (section 2.2). Finally, the whole process of the extraction is converted to web service for online processing. The proposed method is further compared with post-classification comparison method. The performance of annual urban built-up change area extraction was assessed (section 2.3).

\subsection{Spatial-temporal Variation Analysis and Time Series Expression}

The spectral mixing characteristics and spatial-temporal variations of urban built-up area and other land cover types are first explored and analyzed. using spectral data-DN values, spectral index like the normalized difference vegetation index (NDVI), the normalized difference built-up index (NDBI) and the biophysical composition index (BCI) (Deng, et al., 2012). On this basis, inter-annual time series characteristics of urban buildup area and other land cover types is expressed using the index, which can distinguish vegetation and built-up area significantly, as well as reduce the confusion between bare land and built-up area. The time series feature image consists of feature bands, each of which is extracted from a single remotely sensed image by scientific calculation formula of the index. For each pixel of the feature image, there are a series of index values which can reflect land cover types and dynamic change in the time domain

\subsection{Time Series Trajectory Modelling and Change Extraction}

The time series trajectory for the urban built-up area and other land cover types in each pixel is extracted from the time series feature image. Consistency check of the time series trajectory is then conducted. Based on the hypothesis there are few conditions of non-urban built-up area which is transferred to urban built-up area and then transferred back to non- urban built-up area (bare soil), this paper mainly focuses on the urban change of the certain condition, which is only from non-urban built-up area to urban built-up area. The time series trajectory is further reselected according to the hypothesis. Since the single-growth pattern of logistic regression model (Zhang, et al., 2004) is fitted to the urban change in time series feature image, a logistic regression model is used in time series trajectory modelling. Finally, the change time of urban built-up area is calculated based on parameters estimated from time series trajectory model.

\subsection{Processing Service Publication and Information Evaluation}

Referring to the idea of the service-oriented model and based on GlobeLand30 information service platform (www.globeland 30.org), the change extraction approach is converted to web service for online processing, which facilitates users in different regions to meet diverse use and personalized processing needs. In order to evaluate the proposed method, the two-phase comparison method is also used to detect urban built-up area change. The confusion matrix was adopted to assess results with confusion matrix which includes overall accuracy, Kappa coefficient, Producer's accuracy and User's accuracy.

\section{EXPERIMENTAL RESULTS AND DISCUSSION}

\subsection{Study Area and Data}

Beijing, a megacity in China, was selected as the study area for the evaluation of the proposed method. Beijing has experienced a rapid urbanization in the past three decades, resulting in increased infrastructural, housing construction, and urban expansion. The land cover types in the area mainly include water, farmland, grassland, woodland, built-up area and bare land.

Inter-annual time series Landsat TM/ETM+ multispectral images that acquired from 1985-2001 were used in this study. Six reflected spectral bands of Landsat image (i.e., bands 1-5 and 7) with a 30-m spatial resolution for each year were used. The image is free of clouds and with high quality. A relative radiometric correction was conducted for time series expression and analysis, using IRMAD algorithm (Canty, et al., 2004), images were also co-registered with less than 0.5-pixel error. A total of 96 spectral bands were finally used in time series expression. The selected spatial extent of images is $116.066 \mathrm{E}-116.701 \mathrm{E}, 39.661 \mathrm{~N}-40.143$ $\mathrm{N}$, covering an area of approximately $55 \mathrm{~km} \times 55 \mathrm{~km}$ (see Figure 1 ).

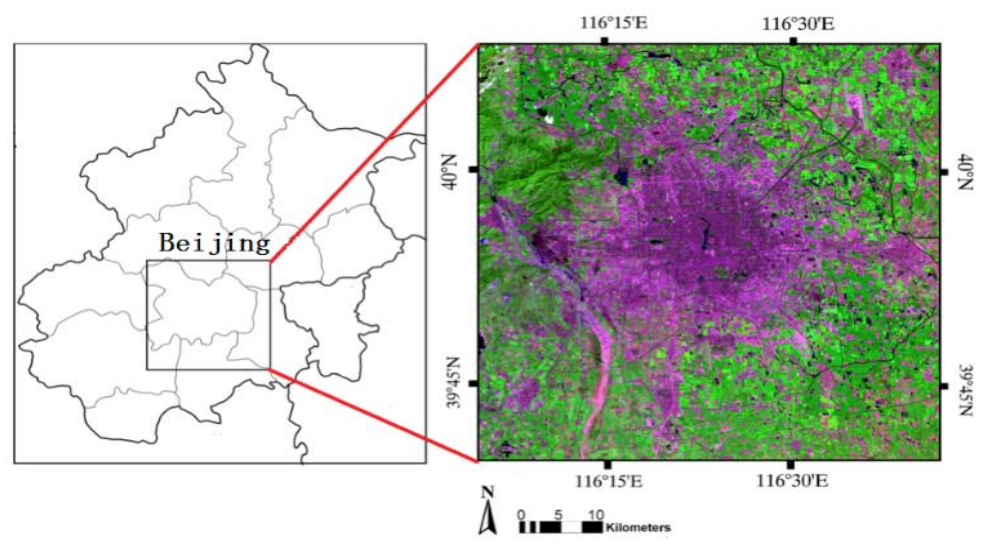

Figure 1. Study area and one Landsat image acquired on 30th Augest,2000, RGB as band 7, 4, 2 


\subsection{Time Series Expression for Urban Built-up Change Area}

Time series analysis is mainly conducted from two aspects, one is the analysis of one land cover type with different time series feature images, another is the comparative analysis between two land cover types with the same time series feature image. Land cover types such as built-up area, vegetation, bare land, and water are analyzed in this study. It is worth noting that by using BCI index, built-up area is significantly distinguished from other land cover types in time series. Thus, the BCI index is finally selected for inter-annual time series characteristics of urban build-up area and other land cover types expression. A BCI time series feature image with 16 feature bands was is extracted from Landsat time series experimental data.

\subsection{Time Series Trajectory Regression Model and Change Extraction}

Time series trajectory consistency check includes outlier value detection and removal, time series trajectory curve filtering. The removal of outliers is mainly aimed at some places where clouds, snow, cloud shadows or other random abrupt changes occur. The feature values of these places are usually abnormal high or low in a time series. In time series trajectory curve filtering, SavitzkyGolay filter (S-G filter) (Savitzky, et al., 1964) which has been widely used is chosen in the study, since it has the advantage of generally keeping the shape of the curve when some small fluctuation points are filtered out.

The time series trajectory curves were further selected based on two conditions. One is the time-BCI Value curve form of time series trajectory should fit to single growth function. And the pixel feature value after abrupt change should in the range of built-up area feature value. Based on selected curves, time series trajectory regression model was built by a logistic algorithm, as in

$$
B C I(t)=\frac{a}{1+e^{b t-c}}+B C I(t-1)
$$

where $t$ represents the year, $a$ represents the change range of in $\mathrm{BCI}$ time series trajectory curve, $b$ represents the change rate, and $c$ represents the change time. According to the formula, the change time for each pixel are determined iteratively.

Table 1 shows urban built-up change area accuracies using the $\mathrm{BCI}$ time series regression model. From the table, on the whole of the assessment parameters, most of the urban built-up change areas were extracted, the overall accuracy is $81.44 \%$, and the Kappa coefficient is $79.20 \%$, the producer's and user's accuracies are mostly over $80 \%$ in each year.

The extraction of urban built-up change area is shown in Figure 2 , it can be seen that urban built-up area is gradually expanded year by year and from the inner city to suburban area. Further analysis of the local areas is carried out. As shown in Figure 3, the highlighted white oval area is mostly of bare land in Figure 3 (a), and mostly of built-up area in Figure 3 (b), it can be found that most of the urban built-up area changes are well extracted by the proposed method. The highlighted yellow oval area is mostly of post-harvest cropland in Figure 3 (a), and cropland in Figure 3 (b), however, the spectral characteristic of the land cover in the Figure 3 (a) is confused with the built-up area. The performance is not be affected by the confusion. This result also validates the performance of the proposed method.

\subsection{Online Processing and Evaluation}

The key open source image processing components, such as GDAL, GSL, Levmar, are the basis of realizing time series change extraction algorithm. The algorithm is transformed into the web service that is supportable for GlobeLand30 information service platform. The DLL (Dynamic Link Library) encapsulation of time series change extraction algorithm was firstly conducted, which provide a solution for the problem of code sharing and invocation across platforms. The W3C WSDL standards were adopted to encapsulate algorithms and publish web service, and finally the TimeSeiresBCI web service is published (see Figure 4).

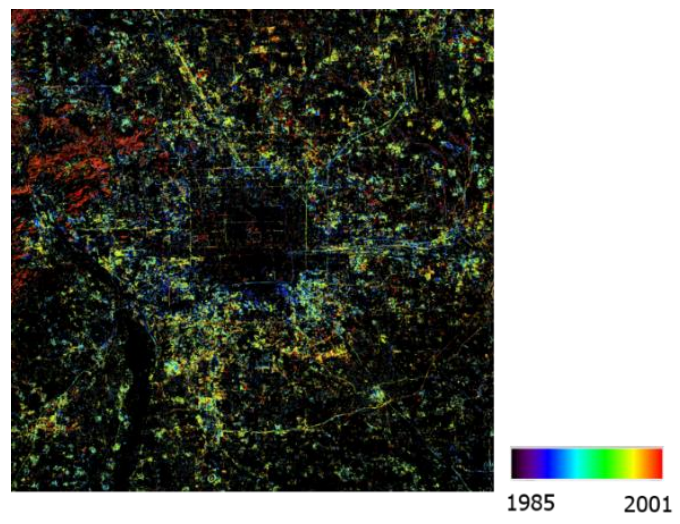

Figure 2. Annual urban built-up change area result

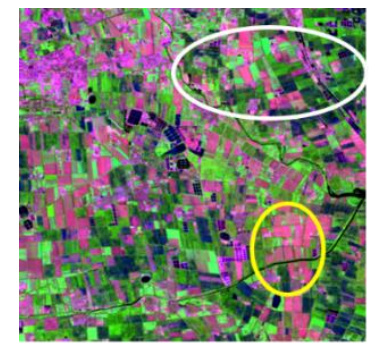

A

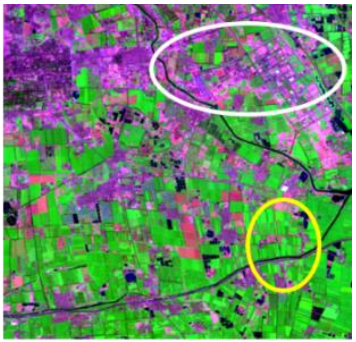

B

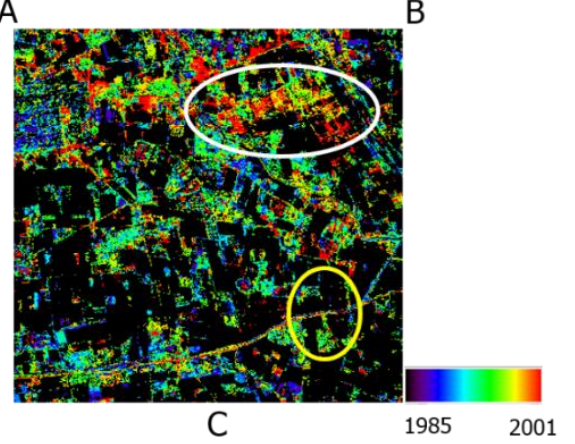

Figure 3. Details of annual urban built-up change area extraction. (a) Portion of one Landsat image acquired in 1985 (DOY: 1985199), RGB as band 7, 4, 2 ; (b) Portion of one Landsat image acquired in 2001 (DOY: 2001243), RGB as band 7, 4, 2; (c) Portion of annual urban built-up change area result

The evaluation was conducted in two aspects, firstly, the time series change extraction algorithm and the post-classification method was compared. It is worth noting that time series change extraction algorithm can significantly reduce confusions of urban and spectral-similar bare land in two-phase comparison method. Then online and offline processing time and convenience were 
compared. It should be noted that the online processing provides a convenient way for common end-users to easily conduct a change extraction approach without desktop-based software, with a small problem of time-cost in data transmission, and the problem will be solved in pace with the development of internet transmission technology.

\section{CONCLUSION}

This paper proposed a new methodology for urban built-up change area extraction using inter-annual time series of Landsat images. The proposed method includes time series characteristics expression, analysis by using spectral data, index and feature, time series trajectory regression model building by using BCI index and logistic algorithm, and processing algorithm conversion as web service for online processing. Urban built-up change area extraction results demonstrated that the proposed method reduced confusion between built-up area and postharvest cropland, as well as built-up area and bare land, and improve the performance with the accuracies of urban built-up change area extraction.

Table 1. accuracies of annual urban built-up change area result (\%)

\begin{tabular}{|c|c|c|c|c|c|c|c|c|c|c|c|c|c|c|}
\hline & 1986 & 1987 & 1988 & 1989 & 1990 & 1991 & 1992 & 1993 & 1994 & 1995 & 1996 & 1999 & 2000 & UA \\
\hline 1986 & 84.09 & 16.92 & & & & & & & 0.37 & & & & & 75.51 \\
\hline 1987 & 11.36 & 73.85 & 5.97 & & & & & & & & & & & 84.21 \\
\hline 1988 & 4.55 & 9.23 & 88.06 & 9.26 & & & & & & & & & & 81.94 \\
\hline 1989 & & & 4.48 & 85.19 & 7.94 & & & & & & & & & 85.19 \\
\hline 1990 & & & 0 & 3.7 & 74.6 & 12.77 & & 0.59 & & & & & & 74.6 \\
\hline 1991 & & & 1.49 & 1.85 & 11.11 & 65.96 & 9.2 & 1.17 & & 0.28 & 0.33 & & & 67.39 \\
\hline 1992 & & & & & 4.76 & 19.15 & 86.5 & 5.28 & & 0 & 0 & & & 78.33 \\
\hline 1993 & & & & & 0 & 2.13 & 3.07 & 87.68 & 15.38 & 0.83 & 0 & & & 85.19 \\
\hline 1994 & & & & & 1.59 & & 1.23 & 4.99 & 84.25 & 2.49 & 0.33 & 0.41 & & 88.12 \\
\hline 1995 & & & & & & & & 0 & & 87.29 & 2.66 & 0.82 & & 96.93 \\
\hline 1996 & & & & & & & & 0 & & 8.56 & 63.79 & 21.81 & 1.4 & 68.82 \\
\hline 1999 & & & & & & & & 0.29 & & 0.28 & 26.25 & 76.13 & 6.05 & 66.31 \\
\hline 2000 & & & & & & & & & & 0.28 & 6.64 & 0.82 & 92.56 & 89.64 \\
\hline PA & 84.09 & 73.85 & 88.06 & 85.19 & 74.60 & 65.96 & 86.50 & 87.68 & 84.25 & 87.29 & 63.79 & 76.13 & 92.56 & \\
\hline
\end{tabular}

OA: overall Accuracy, PA: Producer's accuracy; UA: User's accuracy

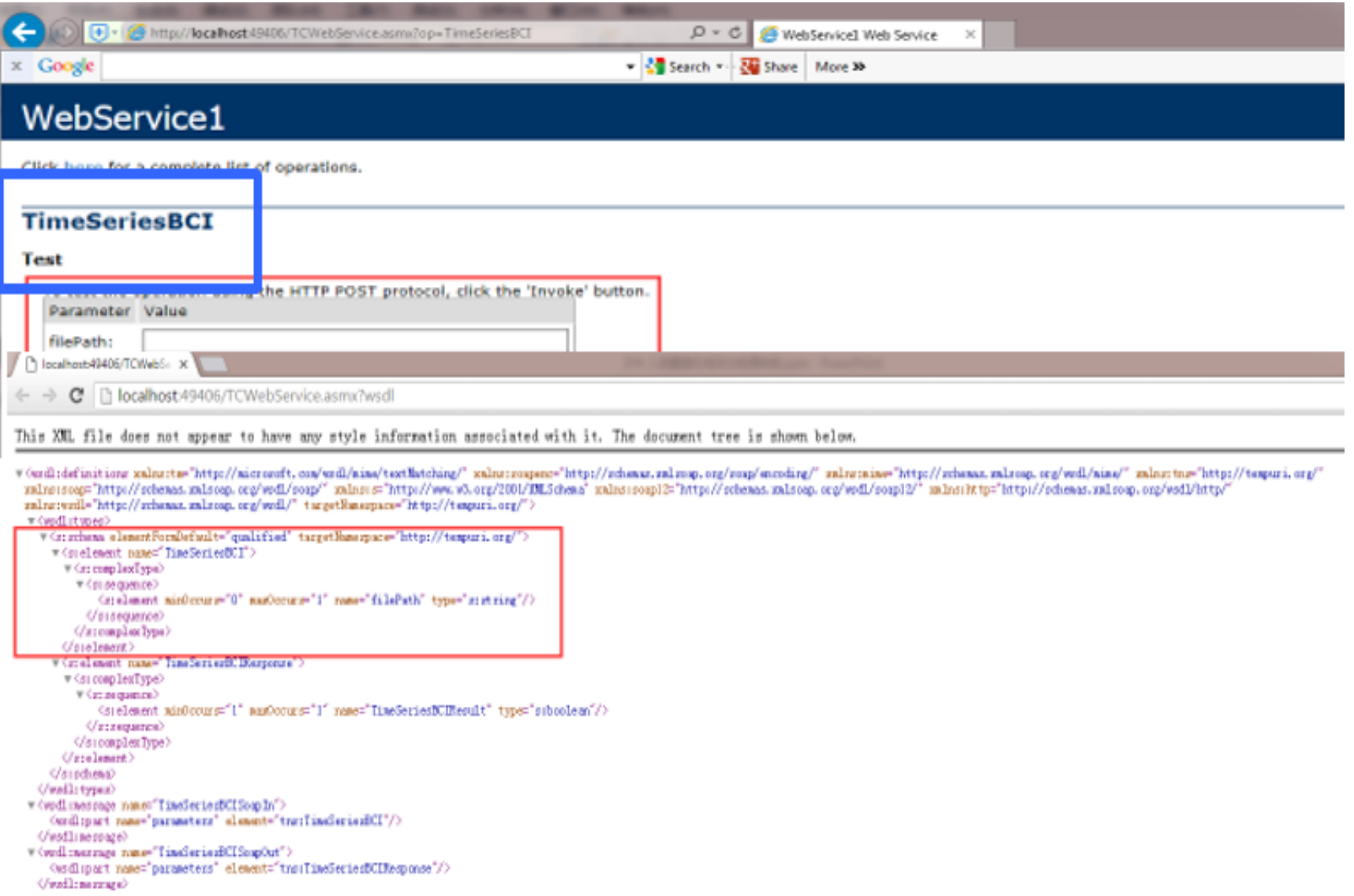

Figure 4. Time Series BCI web service and WSDL file 


\section{REFERENCES}

Aburas, M. M., Ho, Y. M., Ramli, M. F., \& Ash'Aari, Z. H., 2018: Monitoring and assessment of urban growth patterns using spatio-temporal built-up area analysis. Environmental Monitoring \& Assessment, 190(3), 156.

Arsanjani, J. J., Tayyebi, A., \& Vaz, E., 2016: GlobeLand30 as an alternative fine-scale global land cover map: challenges, possibilities, and implications for developing countries. Habitat International. doi: 10.1016/j.habitatint.2016.02.003.

Canty, M.J., Nielsen, A.A., Schmidt, M., 2004: Automatic radiometric normalization of multitemporal satellite imagery. Remote Sensing of Environment, 91(3),441-451.

Chen, J., Dowman, I., Li, S., Li, Z., Madden, M., Mills, J., Paparoditis, N., Rottensteiner, F., Sester, M., Toth, C., 2015: Information from imagery: ISPRS scientific vision and research agenda. ISPRS Journal of Photogrammetry \& Remote Sensing.

Cohen, W. B., Yang, Z., \& Kennedy, R., 2010: Detecting trends in forest disturbance and recovery using yearly Landsat time series: 2. timesync - tools for calibration and validation. Remote Sensing of Environment, 114(12), 2911-2924.

Deng, C., Wu, C., 2012: BCI: A biophysical composition index for remote sensing of urban environments. Remote Sensing of Environment, 127,247-259.

Guo, W., Gong, J.Y., Jiang, W.S., Liu, Y., She, B., 2010: OpenRS-Cloud:A remote sensing image processing platform based on cloud computing environment. Science China Technological Sciences, 53, 221-230.

Hofer, B., 2015: Uses of online geoprocessing technology in analyses and case studies: a systematic analysis of literature. International Journal of Digital Earth, 8, 901-917.

Li, M., Zhu, Z., Huang, C., Shi, H., Lu, H., \& Peng, S., 2009: Assessing rates of forest change and fragmentation in Alabama, USA, using the vegetation change tracker model. Forest Ecology \& Management, 257(6), 1480-1488.

Quan, S., Xiong, B., Xiang, D., \& Kuang, G., 2018: Derivation of the orientation parameters in built-up areas: with application to model-based decomposition. IEEE Transactions on Geoscience \& Remote Sensing, PP(99), 1-17.

Savitzky, A., Golay, M. J., 1964: Smoothing and differentiation of data by simplified least squares procedures. Analytical Chemistry, 36, 1627-1639.

Shen, G., Abdoul, N. I., Zhu, Y., Wang, Z., \& Gong, J., 2017: Remote sensing of urban growth and landscape pattern changes in response to the expansion of chongming island in shanghai, china. Geocarto International, 32(5), 15.

Sumari, N. S., Shao, Z., Huang, M., Sanga, C. A., \& Van Genderen, J. L. , 2017 : Urban expansion: a geo-spatial approach for temporal monitoring of loss of agricultural land. ISPRS - International Archives of the Photogrammetry, Remote Sensing and Spatial Information Sciences, XLII-2/W7, 1349-1355.

Sun, G., Chen, X., Jia, X., Yao, Y., \& Wang, Z., 2017:
Combinational build-up index (cbi) for effective impervious surface mapping in urban areas. IEEE Journal of Selected Topics in Applied Earth Observations \& Remote Sensing, 9(5), 20812092.

Sun, Z., Yue, P., Di, L., 2012: GeoPWTManager: a task-oriented web geoprocessing system. Computers \& Geosciences 47, 34-45. Wu, H., You, L., Gui, Z., Hu, K., Shen, P., 2015: GeoSquare: collaborative geoprocessing models' building, execution and sharing on Azure Cloud. Annals of GIS, 21, 287-300.

Yu, B., Min, T., Wu, Q., Yang, C., Deng, S., \& Shi, K., et al., 2018: Urban built-up area extraction from log- transformed npp-viirs nighttime light composite data. IEEE Geoscience \& Remote Sensing Letters, 15(8), 1279-1283.

Zhang, J., Li, P., Zhang, H., Shu, P., Ming, L., \& Ye, Z., 2016 : An alternative method of urban built-up area extraction using Landsat time series data. Geoscience \& Remote Sensing Symposium.

Zhang, X.Y., Friedl, M.A., Schaaf, C.B., Strahler A.H., 2004: Climate controls on vegetation phenological patterns in northern mid- and high latitudes inferred from MODIS data. Global Change Biology, 10(7), 1133-1145.

Zhu, Z., \& Woodcock, C. E., 2014: Continuous change detection and classification of land cover using all available landsat data. Remote Sensing of Environment, 144(1), 152-171. 